\title{
Evaluación de la erosión hídrica del suelo en la finca "Santos García" ubicada en el cerro Las Mesas, Matagalpa, mediante el uso del radionucleido ambiental ${ }^{137}$ Cesio
}

\section{Maria José Pérez Matus", Karla Hypatia Cruz**, Elias Ramirez Roust- an ${ }^{* * *}$ y Cipriano A. López Lezama**}

Recibido: septiembre de 2014 / aceptado: noviembre de 2014

Se evaluó el proceso de erosión hídrica presente en la finca "Santos García", Ciudad Darío, Matagalpa. Para cuantificar las pérdidas de suelo se empleó la técnica nuclear del ${ }^{137}$ Cesio (como radioisótopo trazador ambiental). A su vez, se analizó la influencia de factores e indicadores que intervienen en el proceso de pérdida de suelo: pendiente y precipitación, textura (método Bouyoucos), color (tabla Munsell), materia orgánica (digestión húmeda con dicromato de potasio), $\mathrm{pH}$ (método Potenciométrico) y capacidad de intercambio catiónico (acetato de amonio pH 7 1N). Los resultados obtenidos reflejan grandes pérdidas de suelo asociadas a las características fisicoquímicas de éste. Se manifiesta la urgente necesidad de implementar medidas de conservación de suelo que permitan la sustentabilidad agrícola en el sitio de estudio.

Palabras clave: erosión hídrica / radioisótopo / fisicoquímicas / conservación

*Universidad Centroamericana, Managua, Nicaragua. Tel. 22696083. Correo electrónico: mjpm199043@ yahoo.es

** Universidad Centroamericana, Managua, Nicaragua. Tel. 22691804. Correo electrónico: karlahc10@ yahoo.es

*** Instituto de Investigación y Desarrollo Nitlapan - Universidad Centroamericana, Managua, Nicaragua. Tel. 88854026. Correo electrónico: ramirezelias05@yahoo.es

**** Facultad de Ciencia, Tecnología y Ambiente - Universidad Centroamericana, Managua, Nicaragua. Tel. 84658415. Correo electrónico: ciprianoagustin@ns.uca.edu.ni 


\section{Introducción}

Las capas superiores del suelo constituyen uno de los recursos naturales más importantes para la existencia humana. Son el sustento de la base alimentaria de cualquier nación. Este delgado manto se pierde con la erosión, uno de los procesos más importantes a tener en consideración en los países tropicales (Mendoza, Brígido, Montalván \& Sánchez, 2006).

La presente investigación pretendió evaluar el proceso de erosión hídrica en la finca "Santos García”, localizada en la comarca Rincón del Diablo del municipio de Ciudad Darío, del departamento de Matagalpa, mediante el uso del radioisótopo trazador Cesio 137 considerando la influencia de factores climáticos, y propiedades físicas y químicas del suelo. La aplicación de la técnica nuclear Cesio137 como radioisótopo trazador ambiental permite cuantificar pérdidas de suelo.

La importancia de esta investigación radica en promover el uso de técnicas precisas para evaluar el proceso de erosión hídrica y así tomar decisiones con mayor grado de precisión basadas en resultados técnicos y no únicamente desde el punto de vista cualitativo.

\section{Diseño metodológico}

\subsection{Descripción de la finca "Santos García"}

En la Finca "Santos García” se siembran granos básicos - maíz y frijol - cuya producción se destina para la alimentación de 30 personas. Además, los agricultores establecen medidas de conservación de suelo, como barreras vivas y muertas en diferentes transeptos, con el fin de reducir el grado de erosión hídrica. La parcela de estudio presenta pendientes de aproximadamente $25 \%\left(14^{\circ}\right)$. Sin embargo, por localizarse esta finca en la falda del cerro Las Mesas, debe considerarse que las inclinaciones de este cerro son superiores al 100\% (45 $)$.

\subsection{Cuantificación de pérdidas de suelo a causa de la erosión hídrica}

\subsubsection{Determinación de puntos de muestreo de suelo}

Con el fin de evaluar el proceso de erosión hídrica y la influencia de los factores de propiedades físicas y químicas del suelo, se realizó la siguiente metodología de muestreo: se determinaron $640 \mathrm{~m}^{2}$ como área de estudio; después se ubicaron dos transeptos de muestreo en sentido de la pendiente, separados entre sí a una distancia de 10 metros. Producto de la implementación en los últimos años de obras de conservación de suelos, en el área de estudio se identificaron (cualitativamente) dos zonas:

- Zona de sedimentación

- Zona de erosión 
Estas zonas están ubicadas en la parte anterior y posterior de las barreras muertas.

En cada transepto se seleccionaron seis puntos de muestreo (12 puntos de muestreo en total - ver Ilustración 1) ubicados de la siguiente forma:

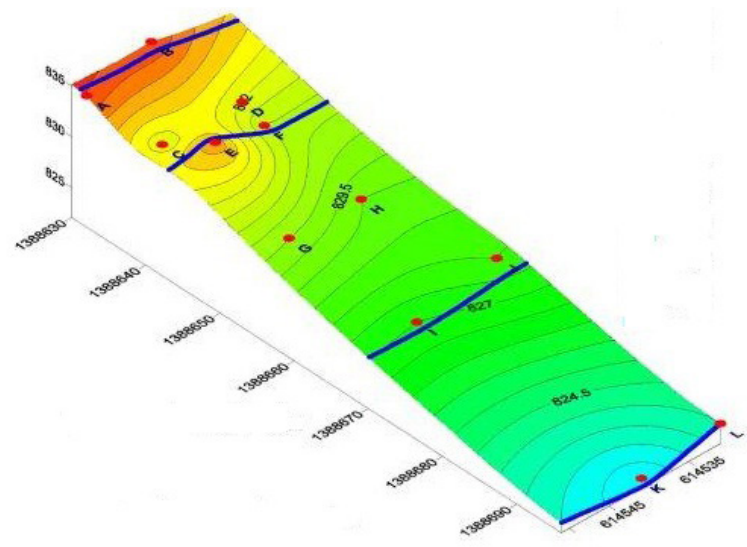

Ilustración 1. Esquema de muestreo

Puntos (A y B): Un metro debajo de la barrera de piedra (se espera erosión).

Puntos (C y D): Un metro antes de la segunda barrera (se espera sedimentación).

Puntos (E y F): Un metro después de la segunda barrera (se espera erosión).

Puntos (G y H): A 15 metros del punto 3 (se espera erosión).

Puntos (I y J): Un metro antes de la barrera 3 (se espera sedimentación).

Puntos (K y L): Un metro antes de la barrera 4 (se espera sedimentación).

Para seleccionar estos puntos de muestreo y determinar de forma cualitativa si serían puntos en los que hay erosión o sedimentación, se consideró la ubicación de las barreras (en la Ilustración 1 las barreras se reflejan con líneas azules).

Al igual que en el sitio de referencia se tomaron cuatro submuestras (36 submuestras en total) por cada punto de muestreo, utilizando cilindros Eijkelkamp de $45 \mathrm{~cm}$ de altura y 5,3 cm de ancho. Luego el cilindro fue extraído con la ayuda de una palanca Eijkelkamp. Las profundidades de muestreo fueron de $0 \mathrm{~cm}-15 \mathrm{~cm}, 15 \mathrm{~cm}$ $30 \mathrm{~cm}$ y de $30 \mathrm{~cm}-45 \mathrm{~cm}$. Estas submuestras fueron homogeneizadas para obtener las respectivas muestras compuestas y representativas de cada punto de muestreo.

\subsubsection{Muestreo de suelo}

El muestro de suelo se realizó el 15 de septiembre de 2012 (temporada de invierno). El suelo se encontraba en estado húmedo. La metodología empleada para cuantificar las pérdidas de suelo calcula la erosión en el tiempo, no siendo relevante (en la obtención de resultados) si el muestreo se efectúa en invierno o verano. 


\section{Identificación de muestras de suelo}

Las muestras de suelo se colocaron en bolsas plásticas de dos libras identificadas según el orden de las letras del alfabeto y la profundidad de muestreo. Se inició con la letra A y se avanzó hasta la L, según el punto del que fue extraída cada muestra.

\section{Pretratamiento de las muestras de suelo}

Al ingresar las muestras al Laboratorio de suelos de la Universidad Centroamericana, se realizó un proceso de limpieza con el propósito de extraer piedras, raíces o cualquier material ajeno al suelo. Seguidamente se realizó pesaje de cada muestra de suelo (Rodríguez, 2002).

El proceso de secado de las muestras de suelo se realizó (según el requerimiento del análisis) con una porción al aire libre y otra porción en el horno a una temperatura de $110^{\circ} \mathrm{C}$ (durante 24 horas) para reducir en lo posible su humedad.

En cuanto a la homogeneización del tamaño, se utilizaron morteros y molinos manuales (según el requerimiento del análisis), limpiándolos entre cada muestra para evitar contaminación.

Como último paso del pre tratamiento, para separar la grava del suelo, se tamizaron las muestras, utilizando tamices de $2 \mathrm{~mm}$.

\section{Medición de actividad del 137Cesio en las muestras de suelo}

La determinación de actividad del ${ }^{137} \mathrm{Cs}$ en las muestras de suelo se efectuó en el Laboratorio de Radioquímica del "Centro para la Investigación en Recursos Acuáticos de Nicaragua” (CIRA) de la Universidad Nacional Autónoma de Nicaragua (UNAN - Managua).

\section{Conversión de unidades de actividad a densidad superficial}

El método de Espectrometría Gamma empleado por el CIRA-UNAN para el análisis de ${ }^{137} \mathrm{Cs}$ en las muestras de suelo expresa unidades de actividad (Bq/ kg) por lo que para convertirlos a unidades de densidad superficial de actividad se utilizó el programa Radionuclides inventories conversion.

\subsubsection{Cuantificación de pérdida de suelo por erosión hídrica}

La cuantificación de pérdida de suelo se realizó mediante el modelo proporcional, contando con las variables requeridas para su aplicación. Dicho modelo se fundamenta en la comparación de la densidad superficial de actividad de ${ }^{137}$ Cesio acumulada en el sitio de referencia con respecto a la densidad superficial de actividad de ${ }^{137}$ Cesio encontrada en el sitio de estudio. Por lo que si hay menor concentración de ${ }^{137}$ Cesio en este último significa que hay pérdida de suelo. Este resultado se expresó en (Carrazana, 2009). 


\section{Análisis de factores climáticos y propiedades físico- químicas}

\subsection{Factores climáticos}

Los datos de precipitación se obtuvieron en el Instituto de Estudios Territoriales (INETER, 2005) y corresponden a la estación meteorológica más cercana al sitio de estudio, la cual se localiza en la comunidad "Dulce Nombre de Jesús".

La pendiente del terreno se determinó con el apoyo de la Coordinación de la carrera de Ingeniería Civil de la Universidad Centroamericana, a través de un grupo de estudiantes y docentes de la carrera.

\subsection{Parámetros físico-químicos}

La determinación de parámetros físico-químicos de las muestras de suelo se realizó en el Laboratorio de Suelos de la Universidad Centroamericana.

En el Cuadro 1 se presentan los métodos empleados para la determinación de parámetros físico-químicos.

Cuadro 1. Métodos de análisis físico-químicos

\begin{tabular}{|l|l|l|}
\hline Parámetro & Tipo & Método \\
\hline Textura & Físico & Método de Bouyoucos \\
\hline Color & Físico & $\begin{array}{l}\text { Tabla Munsell } \\
\text { Método de Digestión Húmeda con } \\
\text { Dicromato de Potasio }\end{array}$ \\
\hline $\mathrm{pH}$ & Químico & Método potenciométrico \\
\hline $\begin{array}{l}\text { Capacidad de Intercambio } \\
\text { Catiónico (CIC) }\end{array}$ & Químico & Método de Acetato de Amonico pH 7 \\
\hline
\end{tabular}

\subsection{Propuestas de medidas de manejo y conservación de suelo}

Considerando los resultados obtenidos del modelo proporcional, gráficos procesados con el software Surffer 9, propiedades físico-químicas del suelo y factores climáticos, se propuso prácticas de conservación de suelo para disminuir la erosión hídrica en el sitio de estudio.

\section{Resultados}

Los resultados obtenidos destacan una alta intensidad de amenaza erosiva y por tanto la urgente necesidad de implementar prácticas de conservación de suelo (Ilustración 2). 


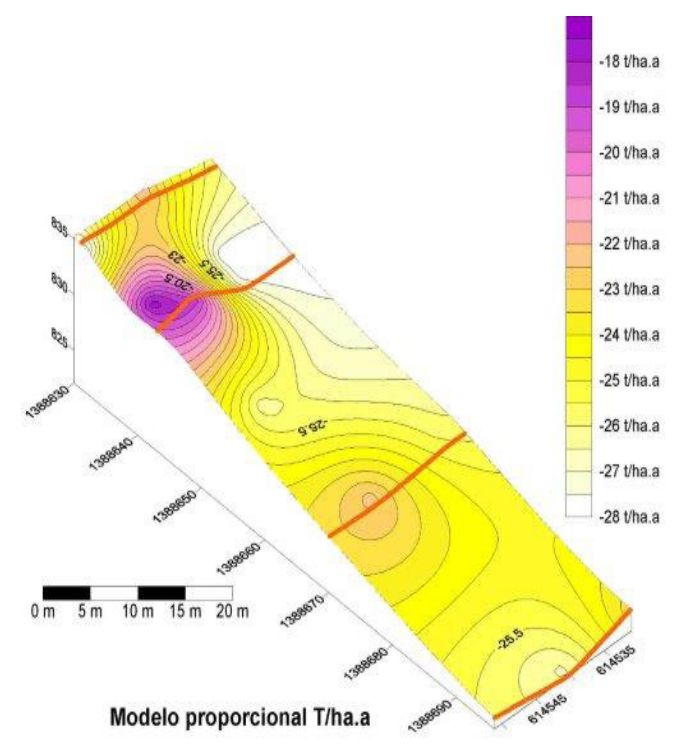

Ilustración 2. Pérdida de suelo en ton/ha. año

\subsection{Análisis de factores que intervienen e indican el proceso de erosión hídrica}

\subsubsection{Textura}

La clase textural predominante del suelo en estudio es de tipo franco, seguida por franco limoso y finalmente por franco arcilloso (Ilustración 3). De lo anterior se determina que la predominancia del limo aumenta la vulnerabilidad erosiva de este suelo. Sin embargo, también es notoria la alta presencia de arena que aunque es resistente al impacto de las gotas de lluvia, cuando se saturan las partículas, su poca plasticidad favorece la escorrentía debido a que su capacidad de retención de agua (oscila del 10\% al 15\%) y de nutrientes es muy baja.

En cuanto a la poca presencia de arcilla, esto hace de la estructura débil en desarrollo debido a la poca estabilidad de los agregados, lo que facilita el arrastre del suelo por la lluvia y el viento.

Ilustración 3. Profundidad vs porcentaje de arena, arcilla y limo

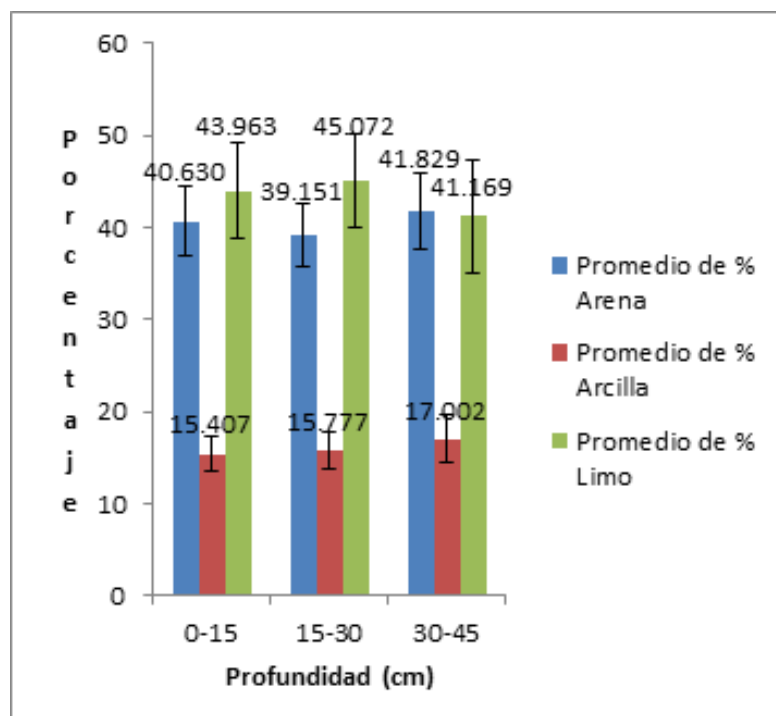




\subsubsection{Color del suelo}

En cuanto al color característico, se aprecia que las profundidades de $0 \mathrm{~cm}$ a $15 \mathrm{~cm}, 15 \mathrm{~cm}$ a $30 \mathrm{~cm}$, y de $30 \mathrm{~cm}$ a $45 \mathrm{~cm}$ (tanto en estado húmedo como en seco) tienen una predominancia de color que está asociada a los niveles del color marrón, que se asocia a los estados iniciales-intermedios de alteración del suelo. A su vez, esto da una idea cualitativa de que el suelo se encuentra con niveles bajos de materia orgánica y fertilidad, factores que se identifican como efectos de pérdida de suelo.

El color negro (estado húmedo del suelo) presente en $P B 15 \mathrm{~cm}$ a $30 \mathrm{~cm}, P L O \mathrm{~cm}$ a $15 \mathrm{~cm}, \mathrm{PL} 15 \mathrm{~cm}$ a $30 \mathrm{~cm}$, de manera cualitativa da una tendencia a considerar que en estos puntos hay altos niveles de materia orgánica (Ovalles, 2003). Sin embargo, en los análisis cuantitativos y la valoración interpretativa realizada para estos puntos se concluyó que son de bajos a muy bajos en contenido de materia orgánica. Por tanto, el color negro (a nivel cualitativo) puede atribuirse a que hay acumulación de $\mathrm{Na}^{+}$y una muy mala condición estructural (propia de suelos erosionados).

\subsubsection{Densidad aparente}

Los resultados apreciados en la Ilustración 4 reflejan que la densidad aparente del suelo aumenta a medida que aumenta la profundidad del horizonte. Sin embargo, en todos los horizontes se aprecian densidades aparentes muy bajas, lo que indica que es un suelo poco compacto y por tanto con una mayor susceptibilidad a la erosión hídrica.

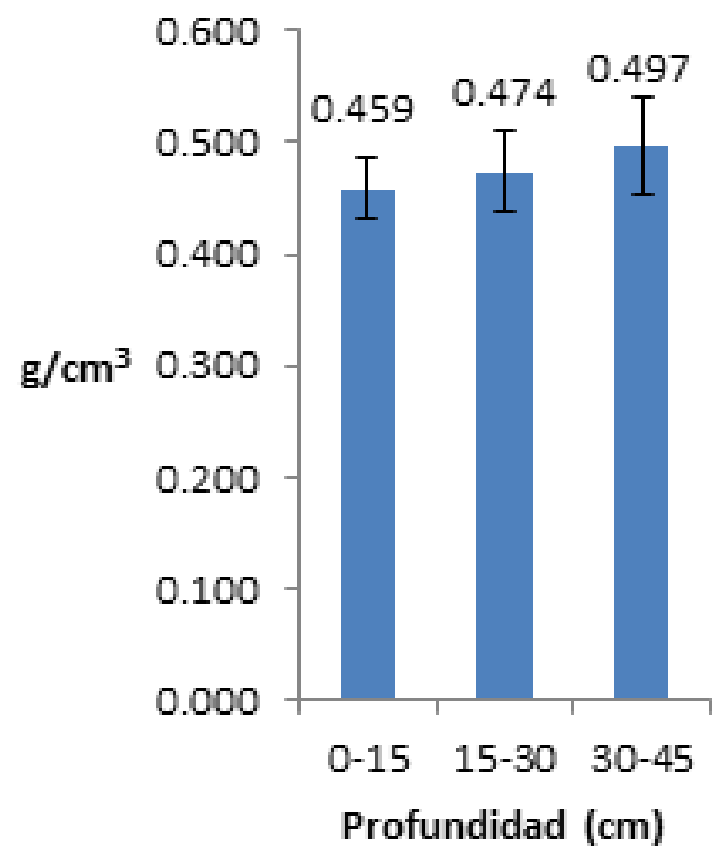

Densidad aparente

Ilustración 4. Profundidad vs densidad aparente 


\subsubsection{Materia orgánica}

La presencia de materia orgánica en este suelo oscila de 0,417\% a 7,091\%, con una valoración interpretativa de baja a muy baja (Meléndez, 2003), (Ilustración 5). La predominancia de materia orgánica aumenta a medida que aumenta la profundidad. Esto puede atribuirse a la poca protección que tiene el suelo, ya que durante cierto tiempo éste se mantiene sin arrope y por tanto la materia orgánica es arrastrada por escorrentías superficiales.

Si se considera el porcentaje de arcilla y el porcentaje de materia orgánica cómo influyentes e indicadores de erosión del suelo, también se evidencia la vulnerabilidad a la erosión hídrica de este suelo. El bajo contenido de materia orgánica y arcilla implica poca formación de terrones y estabilidad de agregados que éstos producen al unirse. Por tanto, su aporte a estabilizar la estructura del suelo es mínimo, aumentando así la erodabilidad.

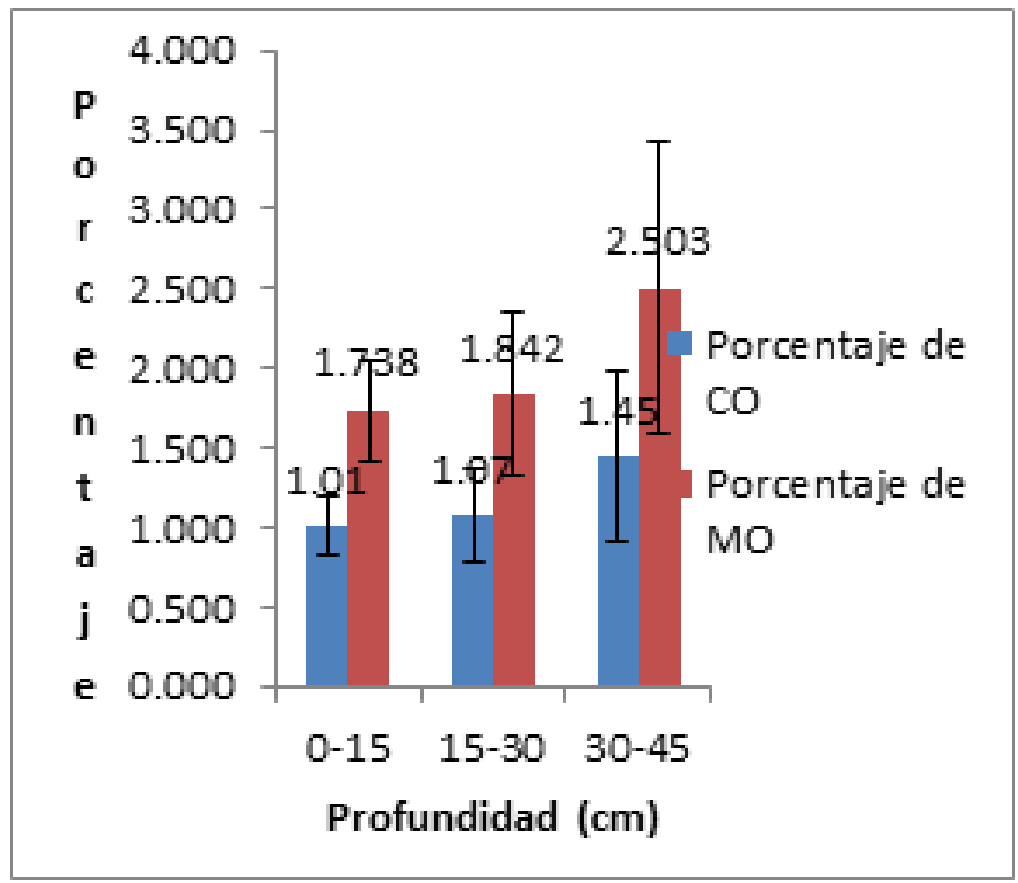

Ilustración 5. Profundidad vs. porcentaje de $\mathrm{CO}$ y MO

\subsubsection{Potencial de Hidrógeno $(\mathrm{pH})$}

Según Yagodin (1986), el rango del potencial de hidrógeno en agua se encuentra de 5,09 a 6,81, mientras que en cloruro de potasio oscila de 4,05 a 5,66 (Ilustración 6). En su mayoría, los puntos muestreados se encuentran en una valoración de $\mathrm{pH}$ neutral, aunque los puntos K 15-30 y L 0-15 presentan acidez. 


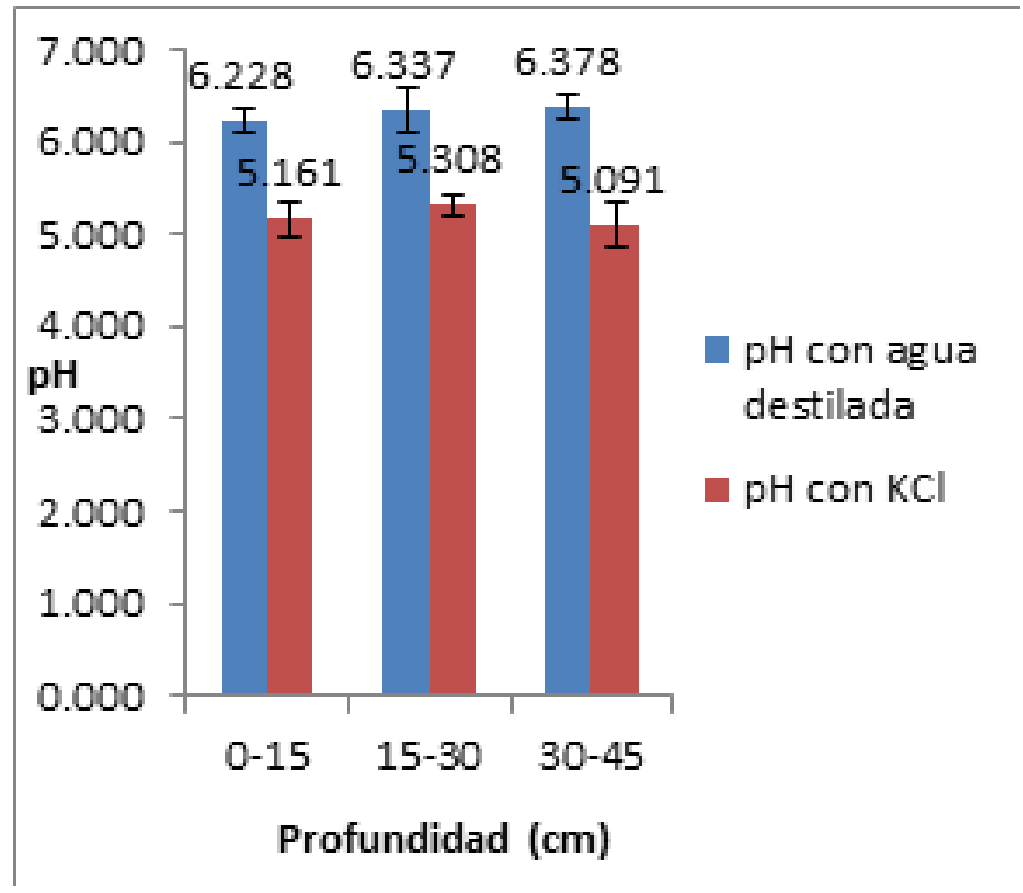

Ilustración 6. Profundidad vs. pH

A medida que aumenta la profundidad se incrementa el $\mathrm{pH}$. Sin embargo, la medición realizada en cloruro de potasio, en la profundidad de $30 \mathrm{~cm}$ a $45 \mathrm{~cm}$, disminuyó con respecto a las profundidades de $0 \mathrm{~cm}$ a $15 \mathrm{~cm}$ y de $15 \mathrm{~cm}$ a $30 \mathrm{~cm}$.

Este suelo se caracteriza como neutral con tendencia a ser ligeramente ácido. Esta tendencia a ser ligeramente ácido puede atribuirse a que el suelo tenga una estructura (considerando el bajo contenido de materia orgánica que muestran los resultados) poco desarrollada. Esto provoca mala aireación, dificultad de laboreo, reducido desarrollo radicular en las plantas, baja permeabilidad y una mayor erodabilidad. Además, es necesario considerar que los suelos ácidos poseen baja capacidad de intercambio de cationes (ver resultados 4.1.6 de Capacidad de Intercambio Catiónico), que también está vinculado a la falta de materia orgánica por la poca capacidad de retención de nutrientes que tiene el suelo.

\subsubsection{Capacidad de Intercambio Catiónico (CIC)}

La capacidad de retención de cationes del suelo aumenta a medida que aumenta la profundidad, encontrándose en un rango de $2 \mathrm{meq} / 100 \mathrm{~g}$ de suelo a $20 \mathrm{meq} / 100$ g de suelo (ver Ilustración 7).

La Capacidad de Intercambio Catiónico se califica como baja. Este comportamiento se puede asociar al bajo contenido de materia orgánica, la textura (predominancia de la presencia de limo) y el $\mathrm{pH}$ con tendencia a ser ligeramente ácido (propio de suelos que carecen de cationes). Su efecto se traduce en baja fertilidad del suelo por la poca capacidad de retención de nutrientes que éste tiene, 
y se evidencia en la poca materia orgánica que tiene este suelo, lo que a su vez es un índice de erosión.

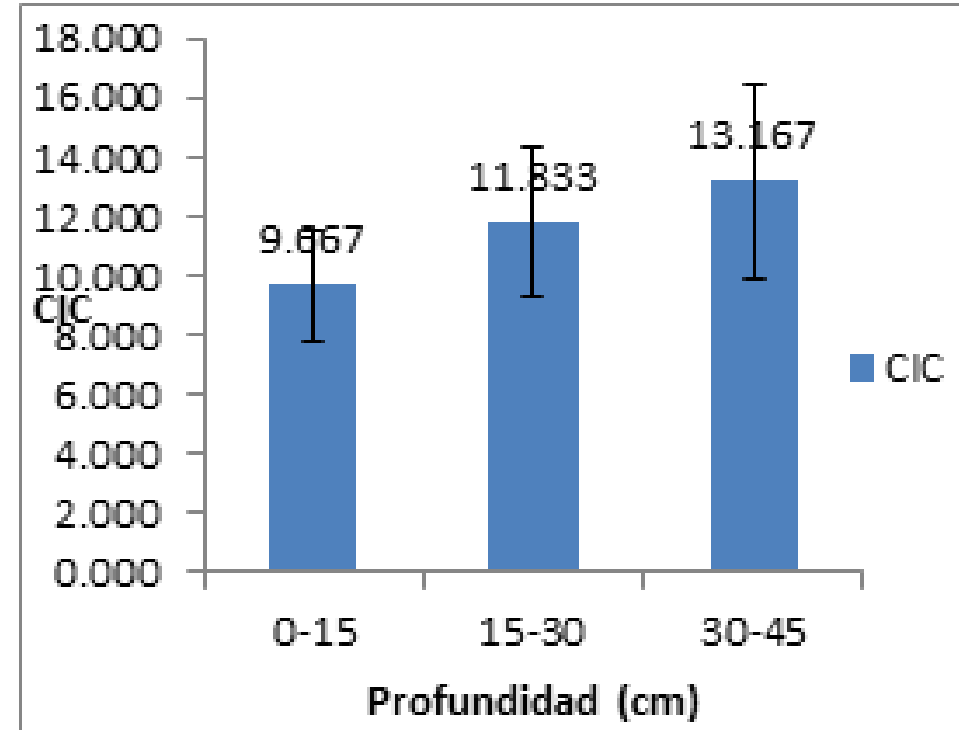

Ilustración 7. Profundidad vs. CIC

\subsubsection{Relieve y pendiente}

El relieve que presenta el sitio de estudio tiene una altura máxima de $835 \mathrm{msnm}$ y una altura mínima de $822 \mathrm{msnm}$. Estas alturas se deben a que la parcela en estudio se encuentra ubicada en la falda del cerro Las Mesas.

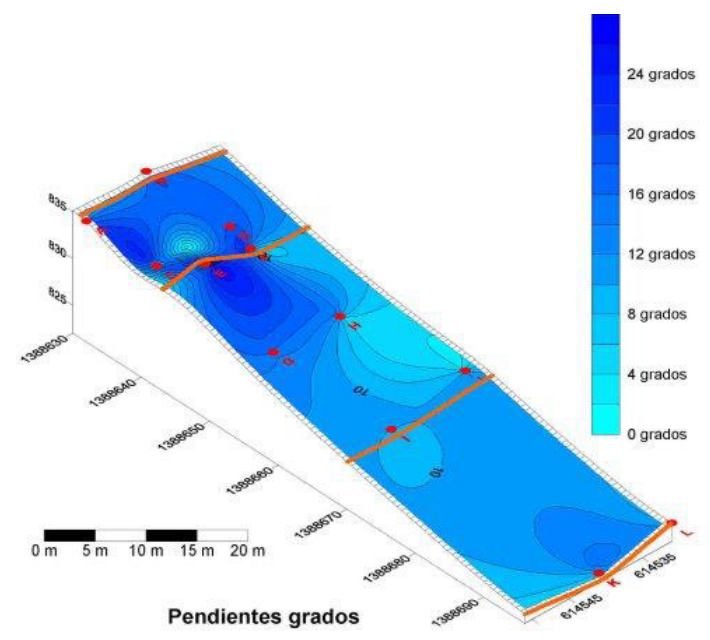

Figura 8. Pendiente del sitio de estudio

El sitio de estudio presenta pendientes que oscilan de $0 \%\left(0^{\circ}\right)$ a $45 \%\left(24^{\circ}\right)$, siendo de $25 \%\left(14^{\circ}\right)$ la pendiente promedio (Ilustración 8). Sin embargo, teniendo presente que el sitio de estudio se encuentra en las faldas del cerro Las Mesas, es 
necesario considerar que la inclinación del cerro supera el $100 \%\left(45^{\circ}\right)$, lo cual es influyente en las pérdidas de suelo que presenta el área de estudio. El grado de estas pendientes está por encima de las pendientes mínimas (5\%-10\%) que deben considerarse como factor erosivo del suelo.

\subsubsection{Clima}

El clima predominante del sitio en estudio es el trópico seco. con precipitaciones que oscilan entre los 0,0 $\mathrm{mm}$ a $842,0 \mathrm{~mm}$ y un promedio mensual de $69,3 \mathrm{~mm}$.

Las lomas (zonas este y oeste del municipio) reciben un poco mas de precipitación, subiendo poco a poco el nivel con variaciones entre $800 \mathrm{~mm}$ y 100 mm de agua y presentan temperaturas un poco más frescas, de entre $22^{\circ} \mathrm{C}$ y $24^{\circ} \mathrm{C}$.

Este clima con altas precipitaciones se ubica por encima del límite inferior, para considerarse como un factor erosivo. Además, estas altas precipitaciones asociadas con el carácter de la roca madre estimulan la diversidad de acidez del suelo, que no favorece la fertilidad e incrementa la erodabilidad.

\subsection{Propuestas de prácticas de conservación de suelo para reducir pérdidas de suelo en el sitio de estudio}

Los resultados de erosión obtenidos denotan la necesidad de aplicar medidas de conservación de suelos. Cabe señalar que actualmente los agricultores de este sitio implementan algunas prácticas agrícolas conservacionistas que disminuyen la erosión hídrica.

Analizando las pérdidas de suelo que está teniendo esta finca y las diversas variables que intervienen e indican el proceso de erosión hídrica (textura, color, densidad aparente, $\mathrm{pH}$, materia orgánica, Capacidad de Intercambio Catiónico, clima, precipitación, pendiente) se propone establecer medidas de conservación de suelos para disminuir la influencia de estos factores en el proceso de erosión hídrica del sitio de estudio:

- Laboreo y siembra en contorno. Por ningún motivo se debe sembrar en el mismo sentido de la pendiente, esta acción aumentaría la velocidad de arrastre de suelo y por tanto se estaría contribuyendo a la erosión hídrica del mismo.

- Arrope del suelo

- Manejo de cobertura. Después de la cosecha, los residuos de ésta (rastrojos) se deben distribuir homogéneamente por toda el área de cultivo.

- $\quad$ Barreras de piedras de alta efectividad en zonas de montañas o barreras vivas (densas hileras de zacate limón que se combina bien con otras técnicas).

- Acequia de ladera

- Formación de tranques perpendiculares a la cárcava. Estos tranques pueden ser convexos o cóncavos, y su profundidad o altura máxima debe ser no menor de $35 \mathrm{~cm}$.

- Diques de piedras con postes que tengan la capacidad de retoñar para fortalecer el muro. 
- Aplicar fertilización orgánica (abonos verdes, compost, lombricompost).

- Rotación de cultivos

- Cultivos intercalados

\section{Conclusiones}

- Las pérdidas de suelo en la finca "Santos García" oscilan aproximadamente de 18,120 a 28,120atribuyéndose estas cuantiosas pérdidas a factores de relieve, precipitación, y propiedades físicas y químicas del suelo. Este valor refleja la poca eficiencia que han tenido las medidas de conservación de suelo que hasta ahora han implementado los agricultores.

- Los valores de pendiente y precipitación oscilan por encima de los rangos mínimos para considerarse como factores influyentes en procesos de erosión hídrica. En cuanto a las propiedades físicas, el tipo del suelo limoso y de color marrón, así como la baja densidad, influyen e indican un proceso de erosión de suelo. Las propiedades químicas valoradas (materia orgánica, pH y Capacidad de Intercambio Catiónico) oscilaron en el rango de baja a muy baja, lo que indica mala estructura en el suelo, poca fertilidad y consecuentemente susceptibilidad a pérdidas de suelo.

- Es necesario implementar medidas que permitan reducir las pérdidas de suelo. Las prácticas de conservación de este valioso recurso deben ser vistas como un sistema en el que se implementan un conjunto de prácticas, ya que implementar una sola práctica no contribuye a resolver el problema de la erosión hídrica.

\section{Agradecimiento}

Se agradece enormemente el apoyo brindado por la Máster Tarsilia Silva, decana de la Facultad de Ciencia, Tecnología y Ambiente - UCA, el doctor Gerd Dercon, asesor técnico de la OIEA, y los doctores cubanos José Luis Peralta y Reynaldo Gil. Se agradece al Laboratorio de Vigilancia Radiológica Ambiental de la Habana Cuba por el análisis de 23 muestras de suelo por espectrometría gamma, así como al Centro de Investigación de Recursos Acuáticos (CIRA) - UNAN MANAGUA por su colaboración en el análisis de 36 muestras por espectrometría gamma.

\section{Referencias bibliográficas}

Carrazana, J. (2009). Introducción a la espectrometría gamma. Curso regional de entrenamiento en muestreo de suelo y espectrometría gamma para estudios de erosión de suelos. La Habana, Cuba, 16 a 27 de noviembre de 2009.

Instituto de estudios territoriales. [INETER]. (2005). Erosión hídrica: Mapas de amenazas y recomendaciones técnicas para su elaboración. Managua: INETER.

Meléndez, G. (2003). Residuos orgánicos y la materia orgánica del suelo. San José: Centro de Investigaciones Agronómicas. Universidad de Costa Rica. Recuperado el 8 de diciembre de 2012, de http://www.cia.ucr.ac.cr/pdf/Memorias/ Memoria\%20Taller\%20Abonos\%200rg\%C3\%A1nicos.pdf. 
Mendoza, L., Brígido, O., Montalván, A. \& Sánchez. (2006). Empleo de la técnica radiactiva del cesio-137 en la determinación de la erosión: Generalidades de la técnica. Centro Agrícola, (2), 87-88.

Ovalles, F. (2003). El Color del Suelo: Definiciones e interpretación. Revista Digital del Centro Nacional de Investigaciones Agropecuarias de Venezuela (3). Recuperado el 8 de diciembre de 2012, de http://sian.inia.gob.ve/repositorio/revistas_ tec/ceniaphoy/articulos/n3/texto/fovalles.html.

Rodríguez, H. (2002). Métodos de análisis de suelos y plantas: Criterios de interpretación. México: Trillas.

Yagodin, B. A. (1986). Agroquimica. Tomo I. Moscú: MIR 